\title{
P16 but not retinoblastoma expression is related to clinical outcome in no-special-type triple-negative breast carcinomas
}

Giuseppe S Bogina ${ }^{1}$, Gianluigi Lunardi ${ }^{2}$, Lisa Marcolini ${ }^{3}$, Matteo Brunelli ${ }^{3}$, Laura Bortesi ${ }^{1}$, Marcella Marconi $^{1}$, Francesca Coati ${ }^{2}$, Matteo Valerio ${ }^{2}$, Massimo Guerriero ${ }^{4}$, Alberto Massocco ${ }^{5}$, Maria C Pegoraro ${ }^{6}$ and Giuseppe Zamboni ${ }^{1}$

${ }^{1}$ Department of Pathology, Sacro Cuore Hospital of Negrar, Verona, Italy; ${ }^{2}$ Department of Oncology, Sacro Cuore Hospital of Negrar, Verona, Italy; ${ }^{3}$ Department of Pathology, University of Verona, Verona, Italy; ${ }^{4}$ Department of Statistic, University of Verona, Verona, Italy; ${ }^{5}$ Department of Surgery, Sacro Cuore Hospital of Negrar, Verona, Italy and ${ }^{6}$ Department of Oncology, ULSS 5 Ovest Vicentino, Vicenza, Italy

Triple-negative breast carcinomas represent a tumor group of pivotal clinical importance given the lack of target therapies. The prognostic significance of triple-negative breast carcinomas remains unclear because of their histological and molecular heterogeneity. Currently, neither prognostic nor predictive factors are available for these tumors. Retinoblastoma $(\mathbf{R b})$ pathway loss has been linked to clinical outcome in various cancer types, including breast cancer. We investigated the association between Rb and p16 protein expression and clinical outcome in no-special-type triple-negative breast carcinomas. Immunohistochemical staining for Rb, p16, p53 and CK5 was carried out on a section from archival specimens of 117 no-special-type triple-negative breast carcinomas. Immunopositive p16 (p16+) and immunonegative $\mathbf{R b}(\mathbf{R b}-)$ staining were seen in $49.5 \%$ and in $\mathbf{2 4 . 8 \%}$ of tumors, respectively. There was an inverse correlation between $\mathbf{p} 16+$ and $\mathbf{R b}-(\boldsymbol{P}<\mathbf{0 . 0 0 1})$. P16 + was correlated with G3 grade $(P<0.001)$, high Ki-67 $(P=0.03)$, p53 overexpression $(P<0.001)$ and CK5 immunopositivity $(P=0.01)$. $R b-$ was not associated with any clinicopathologic variable. Follow-up and therapy data were available in 95 patients. In 20 patients treated with surgery only, neither p16 + nor Rb immunostaining were associated with disease-free survival and overall survival. In 75 patients treated with adjuvant chemotherapy, p16 + was associated with good response to therapy with significant increased disease-free survival $(P=0.001)$ and showed a trend towards a statistical significance for increased overall survival $(P=0.056)$; $\mathbf{R b}$ - were not associated with disease-free survival and overall survival. In multivariate analysis, p16 + was independently associated with disease-free and overall survival, with a hazard ratio of 0.18 (95\% Cl: 0.06-0.51; $\boldsymbol{P}=0.001)$ and 0.21 (95\% Cl: 0.06-0.74; $\boldsymbol{P}=0.015)$, respectively. In patients with no-specialtype triple-negative breast carcinomas, $\mathrm{p} 16+$ is related to good response to adjuvant chemotherapy and can be considered the best surrogate marker for Rb pathway loss.

Modern Pathology (2014) 27, 204-213; doi:10.1038/modpathol.2013.137; published online 26 July 2013

Keywords: chemotherapy; p16; prognosis; Rb; triple-negative breast cancer

The term 'triple-negative breast carcinoma' is commonly used to define breast cancers that are immunohistochemically negative for estrogen (ER) and progesterone receptors (PR) and lacking overexpression and/or amplification of the ERBB2 gene. ${ }^{1-4}$ They account for $10-20 \%$ of all breast

Correspondence: Dr G Bogina, MD, Department of Pathology, Sacro Cuore Hospital, Via Don A Sempreboni 5, 37024 Verona, Negrar, Italy.

E-mail: giuseppe.bogina@sacrocuore.it

Received 9 May 2013; revised 3 June 2013; accepted 12 June 2013; published online 26 July 2013 cancers patients ${ }^{3-5}$ and, generally, have an aggressive clinical course. Moreover triple-negative breast cancers are not a single disease, but a heterogeneous entity having different morphologic and molecular features. In fact, they include special morphologic subtypes, sometimes associated with a better prognosis ${ }^{1,4,6}$ and at least two molecular subtypes of triple-negative breast carcinomas, basallike and non-basal breast carcinomas. ${ }^{3}$ Although basal-like breast carcinomas are currently defined by gene expression profiling, triple-negative breast carcinomas with the expression of basal cytokeratins (CK 5/6, CK14 and CK17) and/or epidermal growth 
factor receptor (EGFR) are often used as an immunohistochemistry surrogate for basal-like breast carcinomas. ${ }^{7}$ Nevertheless, most clinicians use the triple-negative definition for reasons of convenience, as no rigorous staining and scoring protocols have been developed for these basal-like immunohistochemistry markers. ${ }^{3}$ Chemotherapy is currently the mainstay of systemic treatment for triple-negative breast carcinomas because hormonal and HER2-directed therapies are not effective. Currently, neither prognostic nor predictive factors are available to guide treatment decisions. Consequently, in the adjuvant setting, overtreatment with potentially life-threatening side effects or undertreatment without effectiveness is possible. ${ }^{3,5}$ Over the past few years, retinoblastoma (Rb), a tumor suppressor gene that regulates cell-cycle progression, has been associated with disease progression and therapeutic outcome in various cancer types. ${ }^{8}$ Regarding breast carcinomas, $\mathrm{Rb}$ loss is more frequently observed in triple-negative breast carcinomas. ${ }^{7,9,10}$ Molecular Rb pathways' loss were associated with improved response to chemotherapies and resistance to antiestrogen therapies. ${ }^{11-14}$ Unfortunately, immunohistochemistry studies showing a decrease in or lack of Rb protein expression have provided conflicting results concerning prognosis and response to therapies. ${ }^{10,14-20} \mathrm{Rb}$ is inactivated by cyclin-dependent kinase (CDK)4-mediated phosphorylation, and the kinase activity of CDK4 is suppressed by p16INK4a (p16). Because inactivation of $\mathrm{Rb}$ results in the upregulation of p16 expression, high levels of p16 were used as an immunohistochemistry surrogate of $\mathrm{Rb}$ loss, mainly in squamous cell carcinomas harboring high-risk papilloma virus. ${ }^{7}$ Based on morphologic similarity of basal-like breast carcinomas to papilloma virusrelated squamous cell carcinomas of the head and neck, Subhawong et $a l^{7}$ were the first to demonstrate directly that basal-like and other triplenegative breast carcinomas frequently demonstrate $\mathrm{Rb}-/ \mathrm{p} 16$ diffuse positive phenotype.

The aim of this study was to determine the association between $\mathrm{Rb}$ and p16 protein expression and clinical outcome in a well-defined subset of no-special-type triple-negative breast carcinomas.

\section{Materials and methods}

The targets of our study were all no-special-type triple-negative breast carcinomas, diagnosed at the Institute of Pathology of Sacro Cuore Hospital, Negrar, Verona, between January 1998 and December 2010.

Case selection process is shown in Figure 1.

From a dedicated database including all breast cancer, we selected ER/PR $<1 \%$ cases, without HER2 assessment (group 1: 1998-2005) and ER/PR $<1 \%$ and HER2 - (HER2 $=0 / 1+$ immunohistochemistry and $2+$ immunohistochemistry,

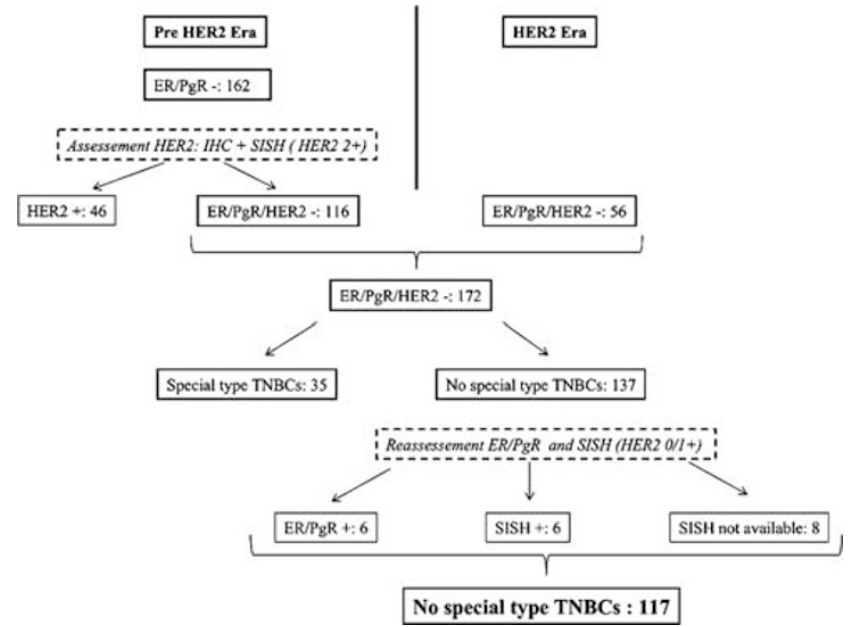

Figure 1 Cases selection algorithm.

silver in situ hybridization non-amplified) (group 2: 2006-2010). Group 1 tissue samples were assessed for HER2 by immunohistochemistry and by silver in situ hybridization in $2+$ immunohistochemistry cases. We obtained 172 triple-negative breast carcinomas diagnosed according to our standard algorithm: 137 were triple-negative breast carcinomas of no special type, whereas 35 were of special type.

Subsequently, no-special-type triple-negative breast carcinomas samples were reassessed for ER/ PR status by immunohistochemistry and by HER2silver in situ hybridization in cases HER2 0/1+ immunohistochemistry. After this reassessment, 6 cases were ER and/or PR $\geq 1 \%$, 6 cases were HER2silver in situ hybridization amplified and for 8 cases silver in situ hybridization was not available. At the end of the analysis, our sample consisted of 117 no-special-type triple-negative breast carcinomas.

Tissue samples were fixed in $10 \%$ buffered formalin for 20-24 h before processing and embedding. Tumors were classified histologically according to the World Health Organization Histological Classification. ${ }^{21}$ Tumor grading was assessed according to Elston and Ellis system. ${ }^{22}$

Data from each patient regarding medical history, results of staging procedures, therapy and follow-up were retrieved. Patients having bilateral breast cancer or others malignant tumors were excluded from the outset.

To evaluate the possible clinical impact of $\mathrm{Rb}$ and p16 expression, we considered disease-free survival, defined as elapsed months since diagnosis of primary tumors to first appearance of any type of relapse (locoregional recurrence or distant metastasis) or censored at the date of last follow-up, and overall survival, defined as elapsed months since diagnosis of primary tumors to death or censored at the date of last follow-up.

Patients having distant metastases at the time of diagnosis were excluded from statistical analysis concerning disease-free survival and overall survival. 
In compliance with the Declaration of Helsinki, this investigation was approved by our reference Ethic Committee.

\section{Immunohistochemistry and Silver In Situ Hybridisation}

From each tumor, the most informative block was selected for immunohistochemistry and silver in situ hybridization. Each block contained normal breast tissue as internal control. For each case, we performed ER, PR, Ki-67, HER2, p16, Rb, p53 and CK5 immunohistochemistry staining and HER2silver in situ hybridization using serial sections from the same paraffin-embedded block. The protocol used for each single antibody is reported in Table 1. The silver in situ hybridisation method, used for ERBB2 gene amplification, was Brightfield Double ISH (VMS-Roche Diagnostics), using INFORM-HER Dual ISH DNA Probe Cocktail, and ultraVIEW AP Red ISH DIG Kit + ultra VIEW SISH DNP Kit detection system.

According to ASCO-CAP guidelines, we defined as positive tumors with ER/PR $\geq 1 \%$. HER2 immunohistochemistry expression was scored according to FDA-approved guidelines as 0 (no staining or weak/moderate, incomplete/complete staining in $\leq 10 \%$ of cells), $1+$ (weak and incomplete staining in $>10 \%$ of cells), $2+$ (weak/ moderate complete staining in $>10 \%$ of cells) and $3+$ (strong, complete staining in $>10 \%$ of cells). For silver in situ hybridization analysis, the ERBB2/ chromosome 17 ratio was calculated and scored as follows: ratio $<2 E R B B 2$ gene not amplified; ratio $\geq 2$ : ERBB2 gene amplified.

Ki-67 was scored evaluating the percentage of positive-stained nuclei (irrespective of staining intensity): based on the median value ( $40 \%$ ), tumors were divided in low and high Ki-67. P16, Rb, p53 and CK5 were scored using a semiquantitative method that takes into account both the percentage and intensity of staining. The percentage of positive tumor cells was established by the assignment of a proportion score: $0=$ none; $1=1-10 \% ; 2=$ $11-30 \% ; 3=31-50 \% ; 4=51-75 \%$; and $5=76-100 \%$. Thereafter, an intensity score, which represented the average intensity of positive tumor cells, was assigned: $0=$ none; $1=$ weak; $2=$ intermediate; and $3=$ strong. The proportion and intensity scores were then multiplied to obtain a total score, which ranged from 0 to 15. The positivity threshold of each biomarker was chosen according to significant values obtained from the literature: $\mathrm{Rb}>07^{7,9,10,16,23}$ p16 $\geq 12 ;^{7,9,23-25}$ p53 $\geq 8 ;^{7,25,26}$ and CK5 $>0 .{ }^{27,28}$

In particular, a p16 score $>12$ correlates with diffuse immunoreactivity in the tumor, as opposed to the more common and less significant patchy immunoreactivity that many cancers show for $\mathrm{p} 16$.

All slides were scored by a single dedicated breast pathologist (GB) who did not have knowledge about patients' outcome.

\section{Statistics}

For statistical analysis, data were imported and merged in STATA/IC for windows version 12.

$\chi^{2}$ or Fisher's exact test were used to compare statistically the categorical variables.

Cumulative incidence of disease-free survival and overall survival in the groups was described by the Kaplan-Meier method and was compared with the use of the log-rank test. Patients alive and not relapsing or alive, regardless of relapsing, were censored at the date of their last follow-up visit for disease-free survival and overall survival, respectively. A two-sided $P$-value $<0.05$ was considered statistically significant. Univariate and multivariate Cox proportional hazard regression model was used to evaluate the independent prognostic relevance of the following factors for disease-free survival and

Table 1 Protocol used for each single antibody

\begin{tabular}{|c|c|c|c|c|c|c|c|c|}
\hline Antibody & Company & Clone & Source & Dilution & Immunostainer & Antigen retrieval & Incubation & Detection \\
\hline Confirm ER Rmab & VMS & SP1 & Rmab & $\mathrm{PD}$ & $\begin{array}{l}\text { BenchMarch XT } \\
\text { VMS }\end{array}$ & $\mathrm{CC} 1 \mathrm{pH} 8.2>$ & $37^{\circ} \mathrm{C} \times 20 \mathrm{~min}$ & $\begin{array}{l}\text { UltraView Universal } \\
\text { Dab Detection }\end{array}$ \\
\hline Confirm PR Rmab & VMS & $1 \mathrm{E} 2$ & Rmab & $\mathrm{PD}$ & $\begin{array}{l}\text { BenchMarch XT } \\
\text { VMS }\end{array}$ & CC1 pH $8.2 \times 30^{\prime}$ & $37^{\circ} \mathrm{C} \times 16 \mathrm{~min}$ & $\begin{array}{l}\text { UltraView Universal } \\
\text { Dab Detection }\end{array}$ \\
\hline Confirm anti-Ki-67 & VMS & K-2 & Mmab & $\mathrm{PD}$ & $\begin{array}{l}\text { BenchMarch XT } \\
\text { VMS }\end{array}$ & CC1 $\mathrm{pH} 8.2 \times 3$ & $37^{\circ} \mathrm{C} \times 16 \min$ & $\begin{array}{l}\text { UltraView Universal } \\
\text { Dab Detection }\end{array}$ \\
\hline Pathway HER-2/neu & VMS & $4 \mathrm{~B} 5$ & Rmab & PD & $\begin{array}{l}\text { BenchMarch XT } \\
\text { VMS }\end{array}$ & CC1 $\mathrm{pH} 8.2 \times 30^{\prime}$ & $37^{\circ} \mathrm{C} \times 16 \min$ & $\begin{array}{l}\text { UltraView Universal } \\
\text { Dab Detection }\end{array}$ \\
\hline Confirm anti-p53 & VMS & D07 & Mmab & $\mathrm{PD}$ & $\begin{array}{l}\text { BenchMarch XT } \\
\text { VMS }\end{array}$ & CC1 $\mathrm{pH} 8,2 \times 30^{\prime}$ & $37^{\circ} \mathrm{C} \times 40 \min$ & $\begin{array}{l}\text { UltraView Universal } \\
\text { Dab Detection }\end{array}$ \\
\hline P16 & SCBT & JC8 & Mmab & $1: 100$ & $\begin{array}{l}\text { BenchMarch XT } \\
\text { VMS }\end{array}$ & CC1 pH $8.2 \times 30^{\prime}$ & $37^{\circ} \mathrm{C} \times 20 \mathrm{~min}$ & $\begin{array}{l}\text { UltraView Universal } \\
\text { Dab Detection }\end{array}$ \\
\hline Cytokeratin 5 & Leica & XM26 & Mmab & $1: 100$ & Bond MaX Leica & ER2 $\mathrm{pH} 6.0 \times 30^{\prime}$ & $\mathrm{RT} \times 15 \mathrm{~min}$ & $\begin{array}{l}\text { Bond Polymer Refine } \\
\text { Detection }\end{array}$ \\
\hline RB gene protein & Leica & $13 \mathrm{~A} 10$ & Mmab & $1: 50$ & Bond MaX Leica & ER2 $\mathrm{pH} 9.0 \times 30^{\prime}$ & $\mathrm{RT} \times 15 \mathrm{~min}$ & $\begin{array}{l}\text { Bond Polymer Refine } \\
\text { Detection }\end{array}$ \\
\hline
\end{tabular}

Abbreviations: Leica, Leica Biosystems, Newcastle, UK; Mmab, mouse monoclonal antibody; RB, retinoblastoma; Rmab, rabbit monoclonal antibody; SCBT, Santa Cruz Biotechnology, Santa Cruz, CA, USA; VMS, Ventana Medical Systems, Tucson AZ, USA. 
overall survival: age ( $\leq 45$ years $v s>45$ years); tumor size (T1 vs T2-T4); node status (negative vs positive $\leq 3$ vs positive $>3$ ); histological grade (grade 2 vs grade 3); vascular invasion (absent vs present); Ki-67 immunostaining percentage $(\leq 40 \%$ vs $>40 \%$ ); p16 (<12 vs $\geq 12)$; and $\mathrm{Rb}(0$ vs $\geq 1)$ immunostaining score. In multivariate analysis, all variables were initially included into the model and then removed by backward stepwise selection if their $P$-value was $>0.05$. To verify the proportional hazard assumption, estat phtest (test based on Shoenfeld residuals) and linktest (test for model goodness of fit) STATA commands were used.

\section{Results}

From reassessment of ER, PR and HER2, as described above, we obtained 152 triple-negative breast carcinomas: 117 of no special type and 35 of special type (14 apocrine, 10 metaplastic, 5 lobular, 3 adenoid cystic and 3 medullary carcinoma). We focused our analysis on 117 no-special-type triplenegative breast carcinomas. Their clinicopathologic features and therapy data are summarized in Table 2 .

\section{Association of P16 and Rb Expression with Pathologic Features}

P16 immunopositivity (p16 + ) was seen in 58 of 117 triple-negative breast carcinomas (49.5\%), whereas Rb immunonegativity $(\mathrm{Rb}-$ ) was seen in 29 of 117 triple-negative breast carcinomas (24.8\%) (Table 3). There was a clear inverse correlation between p16+ and $\mathrm{Rb}-(P<0.001)$ : tumors with p16 strong immunostaining were generally devoid of $\mathrm{Rb}$ immunostaining in the tumor compartment, although stroma and lymphocytes were positive (Figures 2a-c), whereas tumors with p16 absent/low immunostaining were Rb immunopositive (Figures 2d-f). P16 + was correlated with G3 grade $(P<0.001)$, high Ki-67 $(P=0.03)$, p53 overexpression $(P<0.001)$ and CK5 immunopositivity $(P=0.01)$. Other clinicopathologic variables, such as age, tumor size, vascular invasions and lymph node status, were not associated with $\mathrm{p} 16+$. Rb - was not associated with any clinicopathologic variables.

\section{Association of P16 and Rb Expression with Disease Outcomes}

Disease-free survival and overall survival analysis was possible in 95 out of 117 patients: in 14, followup and/or therapy was unknown, whereas 8 were excluded because they had distant metastases at the time of diagnosis. Twenty out of 95 patients were treated with surgery only, whereas 75 patients were treated with surgery and adjuvant chemotherapy. Disease progression occurred in 27 patients: 6
Table 2 Clinicopathologic features of 117 TNBCs of no special type

\begin{tabular}{|c|c|}
\hline & Patients: 117 \\
\hline Median age (years) (range) & $59(26-97)$ \\
\hline \multicolumn{2}{|l|}{ Tumor size (mm) } \\
\hline$\leq 20$ & 60 \\
\hline$>20$ & 57 \\
\hline \multicolumn{2}{|l|}{ Tumor grading } \\
\hline G1 & 0 \\
\hline G2 & 12 \\
\hline G3 & 105 \\
\hline \multicolumn{2}{|l|}{ Vascular invasions } \\
\hline Yes & 29 \\
\hline No & 83 \\
\hline Unknown & 5 \\
\hline \multicolumn{2}{|l|}{ No. of positive nodes } \\
\hline 0 & 67 \\
\hline $1-3$ & 23 \\
\hline$\geq 4$ & 18 \\
\hline Unknown & 9 \\
\hline \multicolumn{2}{|l|}{ Ki-67 (\%) } \\
\hline$\leq 40$ & 70 \\
\hline$>40$ & 47 \\
\hline \multicolumn{2}{|l|}{ Chemotherapy } \\
\hline Yes & 83 \\
\hline No & 27 \\
\hline Unknown & 7 \\
\hline \multicolumn{2}{|l|}{ Chemotherapeutic regimens } \\
\hline $\mathrm{CMF}$ & 15 \\
\hline $\mathrm{CMF}+\mathrm{A}$ & 11 \\
\hline $\mathrm{CMF}+\mathrm{A}+\mathrm{T}$ & 11 \\
\hline FEC & 17 \\
\hline $\mathrm{FEC}+\mathrm{T}$ & 16 \\
\hline $\mathrm{A}+\mathrm{C}$ & 9 \\
\hline Other & 4 \\
\hline
\end{tabular}

Abbreviations: A, anthracycline; C, cyclophosphamide; CMF, cyclophosphamide; FEC, 5-fluorouracil, epirubicin, cyclophosphamide; T, taxane; TNBCs, triple-negative breast carcinomas, methotrexate, 5-fluorouracil.

experienced locoregional recurrence, 11 distant metastasis and 10 both locoregional recurrence and distant metastasis. The median relapsing time was 23 months. Twenty patients were dead at the time of analysis. The median time to death was 37.5 months. Patients alive and disease free at the time of analysis had a median follow-up of 80 months. In patients treated with surgery only, neither p16 + nor $\mathrm{Rb}$ - immunostaining were associated with diseasefree survival and/or overall survival $(P=0.11$ and 0.40 for $\mathrm{p} 16$ and $P=0.32$ and 0.67 for $\mathrm{Rb}$, respectively) (Figure 3). In patients treated with surgery and adjuvant chemotherapy, p16 + was associated with good response to therapy: Kaplan-Meier analysis showed a statistically significant increased disease-free survival $(P=0.001)$ and a trend towards a statistical significance for increased overall survival $(P=0.056)$ (Figures 4a and b). Conversely, $\mathrm{Rb}-$ was not associated with disease-free survival and 
Table 3 P16 and Rb expression in 117 TNBCs of no special type in relation to clinicopathologic variables

\begin{tabular}{|c|c|c|c|c|c|c|}
\hline & $\begin{array}{c}\text { P16- } \\
\text { (59 patients) }\end{array}$ & $\begin{array}{c}\text { P16+ } \\
\text { (58 patients) }\end{array}$ & $\mathrm{P}$-value & $\begin{array}{c}R b- \\
\text { (37 patients) }\end{array}$ & $\begin{array}{c}R b+ \\
\text { (80 patients) }\end{array}$ & $\mathrm{P}$-value \\
\hline \multicolumn{7}{|l|}{ Age (years) } \\
\hline$\leq 45$ & 9 & 12 & 0.47 & 9 & 12 & 0.29 \\
\hline$>45$ & 50 & 46 & & 28 & 68 & \\
\hline \multicolumn{7}{|c|}{ Tumor size (mm) } \\
\hline$\leq 20$ & 34 & 26 & 0.19 & 17 & 43 & 0.55 \\
\hline$>20$ & 25 & 32 & & 20 & 37 & \\
\hline \multicolumn{7}{|c|}{ Vascular invasions } \\
\hline Yes & 16 & 13 & 0.52 & 6 & 22 & 0.16 \\
\hline No & 39 & 44 & & 31 & 53 & \\
\hline Unknown & 4 & 1 & & 0 & 5 & \\
\hline \multicolumn{7}{|c|}{ No. of positive nodes } \\
\hline 0 & 30 & 37 & 0.36 & 22 & 45 & 0.64 \\
\hline $1-3$ & 14 & 9 & & 8 & 15 & \\
\hline$\geq 4$ & 10 & 8 & & 4 & 14 & \\
\hline Unknown & 5 & 4 & & 3 & 6 & \\
\hline \multicolumn{7}{|l|}{ Tumor grade } \\
\hline G2 & 12 & 0 & $<0.001$ & 3 & 9 & 0.75 \\
\hline G3 & 47 & 58 & & 34 & 71 & \\
\hline \multicolumn{7}{|l|}{ Ki-67 (\%) } \\
\hline$\leq 40$ & 42 & 28 & 0.014 & 18 & 52 & \\
\hline$>40$ & 17 & 30 & & 19 & 28 & \\
\hline \multicolumn{7}{|l|}{$p R b$} \\
\hline Negative & 6 & 31 & $<0.001$ & & & \\
\hline Positive & 53 & 27 & & & & \\
\hline \multicolumn{7}{|l|}{ P53 } \\
\hline Negative & 38 & 16 & $<0.001$ & 15 & 39 & 0.43 \\
\hline Positive & 21 & 42 & & 22 & 41 & \\
\hline \multicolumn{7}{|l|}{ CK5 } \\
\hline Negative & 28 & 14 & 0.012 & 6 & 24 & 0.17 \\
\hline Positive & 31 & 44 & & 31 & 56 & \\
\hline
\end{tabular}

Abbreviation: TNBCs, triple-negative breast carcinomas.

p16 positive: IHC score $\geq 12$; Rb and CK5 positive: IHC score $\geq 1$; p53 positive: IHC score $\geq 8$.

overall survival ( $P=0.66$ and 0.89 , respectively) (Figures 4c and d).

Results of multivariate Cox proportional hazards regression analysis, adjusted for age, tumor size, node status, histological grading, vascular invasion, $\mathrm{Ki}-67, \mathrm{Rb}$ and p16 immunostaining are reported in Table 4. In this model, p16 + was independently associated with disease-free survival and overall survival, with a hazard ratio of 0.18 (95\% CI: 0.06-0.51; $P=0.001$ ) and 0.21 (95\% CI: $0.06-0.74$; $P=0.015)$, respectively.

\section{Discussion}

RB1 gene, located on chromosome 13 (13q14), encodes a nuclear phosphoprotein that regulates cell-cycle progression through the G1- to-S-phase transition. In quiescent cells, Rb is hypophosphorylated and, assembling transcriptional repressor complexes on the promoters of E2F-regulated genes, blocks cell-cycle progression. In cells entering cell cycle, extracellular signals induce the expression of D-type cyclins, which bind to and activate cyclindependent kinases (CDK4 and CDK6); these complexes in turn lead to the phosphorylation of $\mathrm{Rb}$ and its dissociation from E2F family members that then transcriptionally activate many genes required for cell-cycle progression. ${ }^{29} \mathrm{P} 16$, the principal member of the INK4 family of CDK inhibitors, is codified by the CDKN2A gene localized on chromosome 9p21. The binding of p16 to CDK4/6 inhibits CDK4 and CDK6, retaining $\mathrm{Rb}$ in its hypophosphorylated status, preventing cell-cycle progression from G1 to $\mathrm{S}$ phase. Elevated expression of p16 is a strong mechanism of inhibition of proliferation and cellular senescence induction, resulting in tumor development block. On the other hand, p16 overexpression is observed in a number of cancers with inactivated $\mathrm{Rb}$ : a cell with a compromised $\mathrm{Rb}$ pathway will initiate a regulatory-induced overexpression of p16 because of negative feedback regulation. 

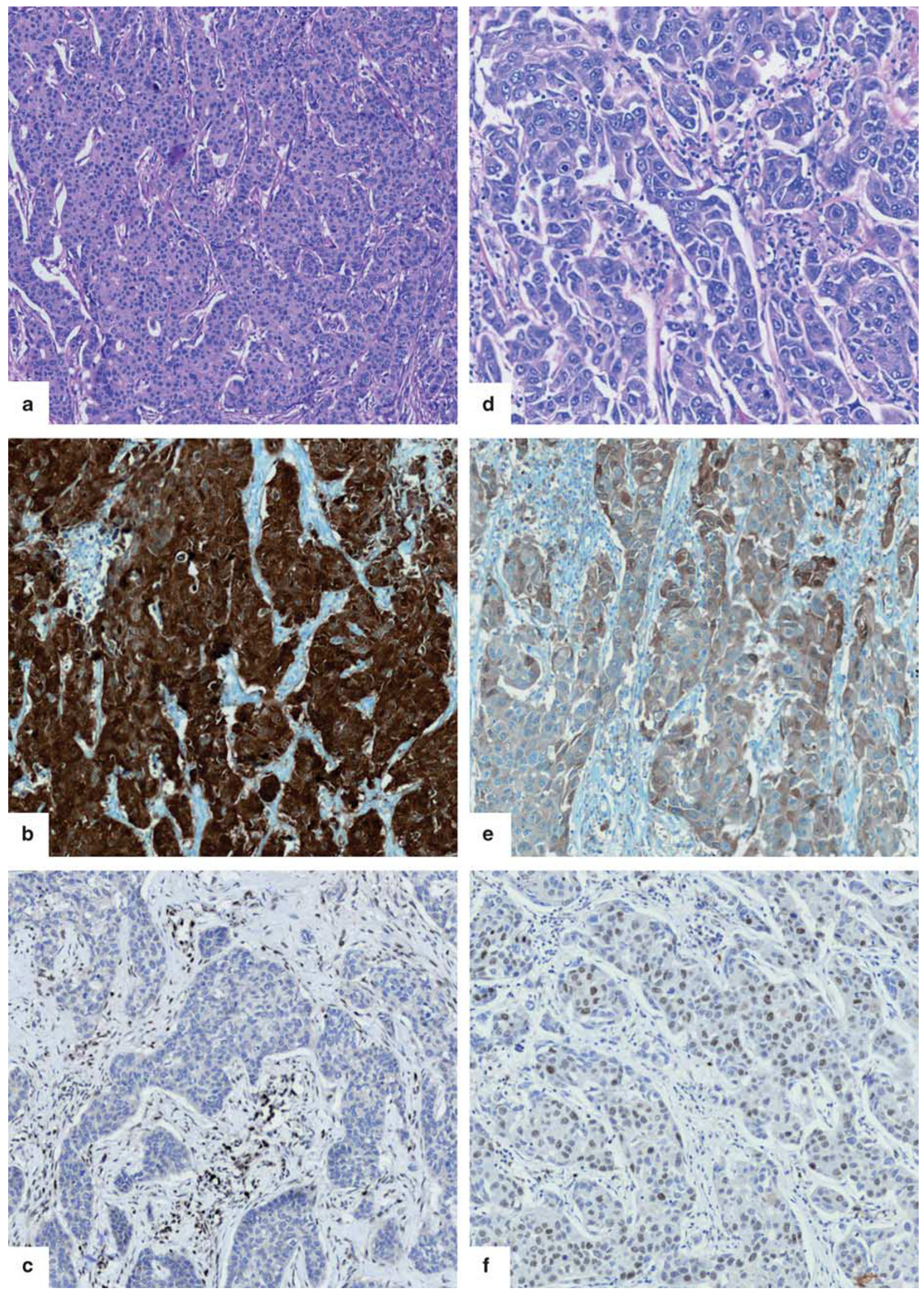

Figure 2 Two different examples of high-grade, no-special-type triple-negative breast carcinomas. Case 1 (a-c): Hematoxylin-eosin $(\times 20)(\mathbf{a})$ with strong immunoreactivity for p16 (b) and negativity for Rb (stromal and lymphoid control cells positive) (c). Case $2(\mathbf{d}-\mathbf{f})$ : Hematoxylin-eosin $(\times 20)(\mathbf{d})$ with p16 low $(\mathbf{e})$ and $\mathrm{Rb}+(\mathbf{f})$ immunostaining. 


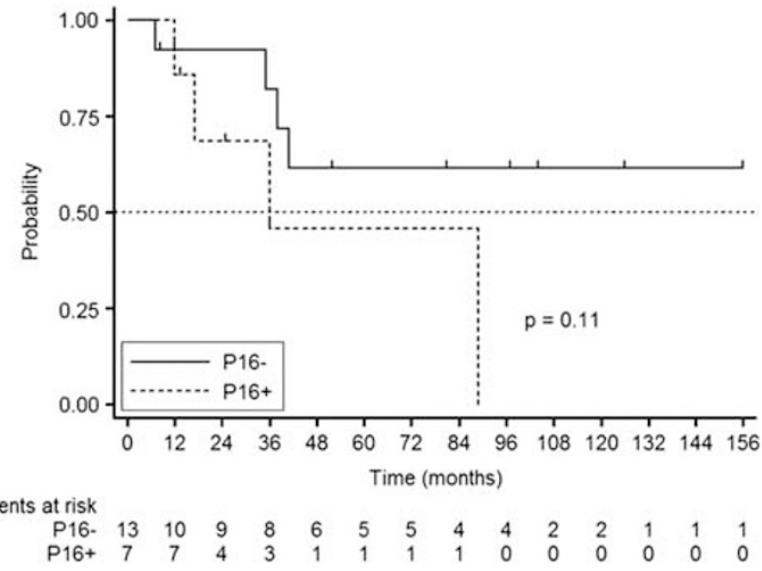

c

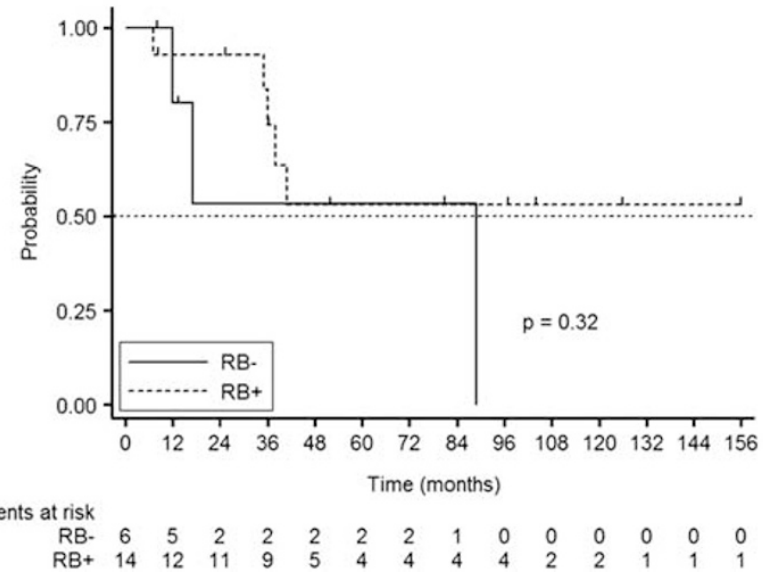

b

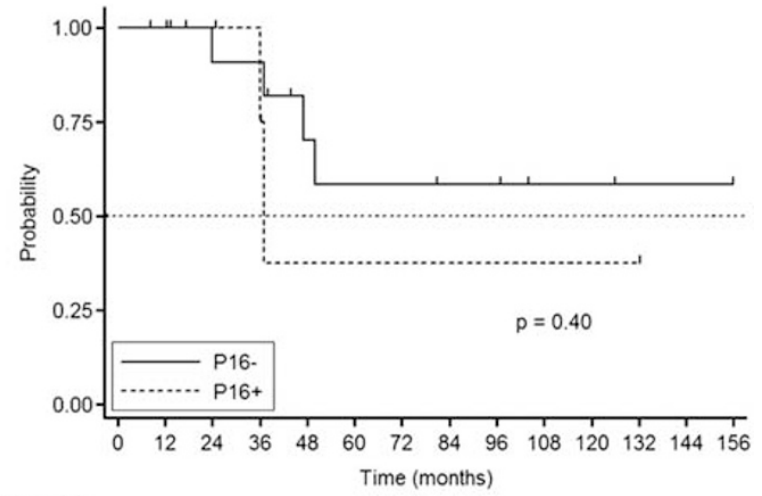

Patients at risk

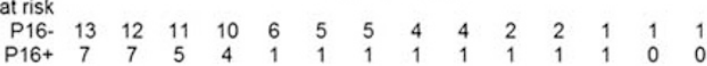

d

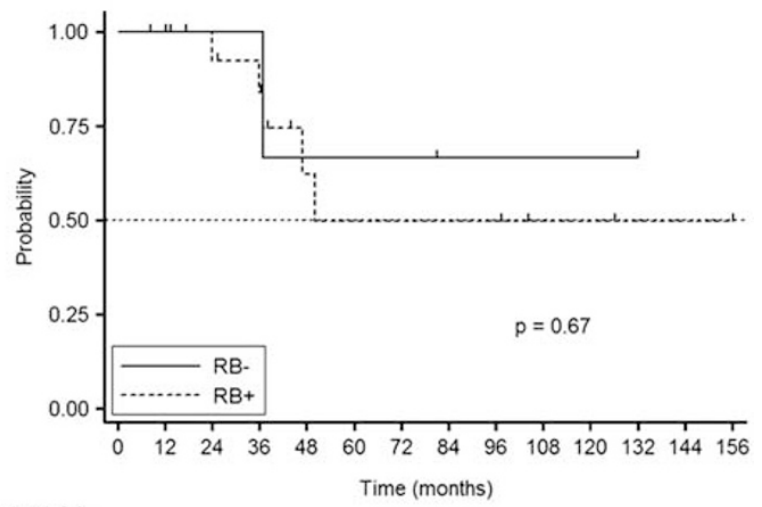

Patients at risk

$\begin{array}{ccccccccccccccc}\mathrm{RB}- & 6 & 5 & 3 & 3 & 2 & 2 & 2 & 1 & 1 & 1 & 1 & 1 & 0 & 0 \\ \mathrm{RB}+ & 14 & 14 & 13 & 11 & 5 & 4 & 4 & 4 & 4 & 2 & 2 & 1 & 1 & 1\end{array}$

Figure 3 Disease outcome related to p16 and Rb protein expression in patients treated with surgery only. (a and b) P16 + was not associated with disease-free survival (a) and overall survival (b). (c and d) Rb - was not associated with disease-free survival (c) and overall survival (d).

Elevated expression of p16, in conjunction with a high proliferative index, is believed to be indicative of $\mathrm{Rb}$ functional loss. ${ }^{23,30}$ In recent molecular studies, $\mathrm{Rb}$ loss signature has been linked to poor prognosis in breast cancer patients receiving adjuvant endocrine therapy and to good prognosis in patients receiving chemotherapy. ${ }^{11-14} \mathrm{Rb}$ immunohistochemistry studies have provided conflicting results concerning prognosis and response to therapies, and also because of the use of different immunohistochemistry cutoff. ${ }^{10,14-20}$ The results reported by Derenzini et $a l^{16}$ indicated that only the absence but not hyperphosphorylation of $\mathrm{Rb}$ protein predicts clinical outcome. However, it should be noted that immunohistochemistry expression of $\mathrm{Rb}$ has provided contradictory interpretations concerning correlation with $R B 1$ gene status. Herschkowitz et $a l^{9}$ showed that $\mathrm{Rb}$ loss of heterozygosity, occurring at a frequency of $72.2 \%$ in basal-like breast carcinomas, did not correlate with Rb absent immunostaining, but it significantly correlates with p16 strong immunostaining.

Only a few studies have investigated the correlation between p16 and clinical outcome in breast cancers. In these studies, strong immunoexpression of p16 was associated with improved response to neoadjuvant chemotherapy in invasive breast cancer. $^{24}$

Despite $\mathrm{Rb} / \mathrm{p} 16$ pathway perturbation being a more common event in triple-negative breast carcinomas, ${ }^{7,9,10}$ only a few studies investigated $\mathrm{Rb} / \mathrm{p} 16$ correlation with clinical outcome in this subset of tumors. ${ }^{10,24}$ In triple-negative breast carcinomas, there are not established markers predicting chemotherapy response, although it is very heterogeneous. ${ }^{14}$ This heterogeneity is also because of the presence of some special histotypes of triplenegative breast carcinomas, which are associated with different prognosis. ${ }^{1,4,6}$

The aim of our study was to evaluate the association between p16 and Rb immunostaining and clinical outcome in a well-defined subset of no-special-type triple-negative breast carcinomas.

In agreement with other authors, ${ }^{7,9,14}$ we confirmed an inverse relationship between p16 and Rb protein expression: tumors with p16 absent/low immunostaining were $\mathrm{Rb}$ immunopositive, whereas those with p16 strong immunostaining were 
a

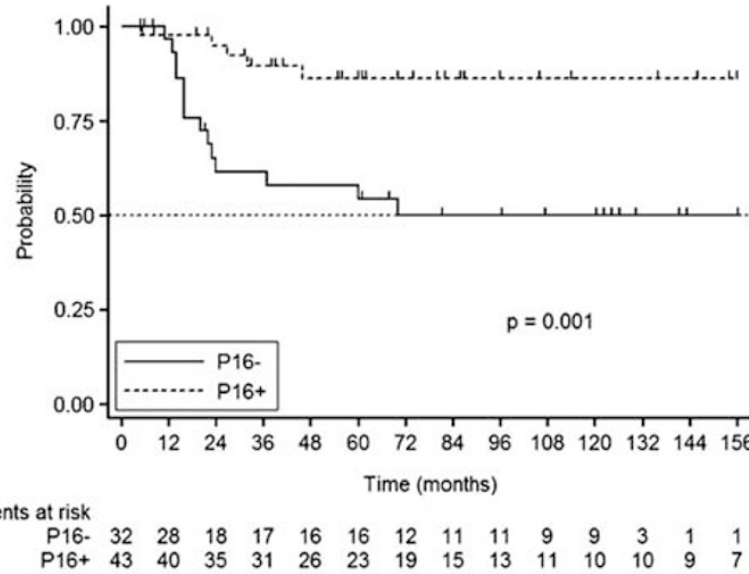

C

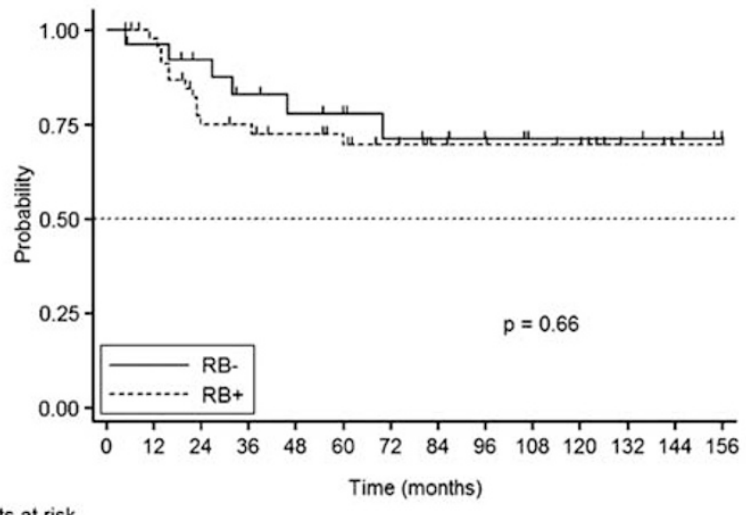

Patients at risk

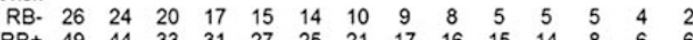

b

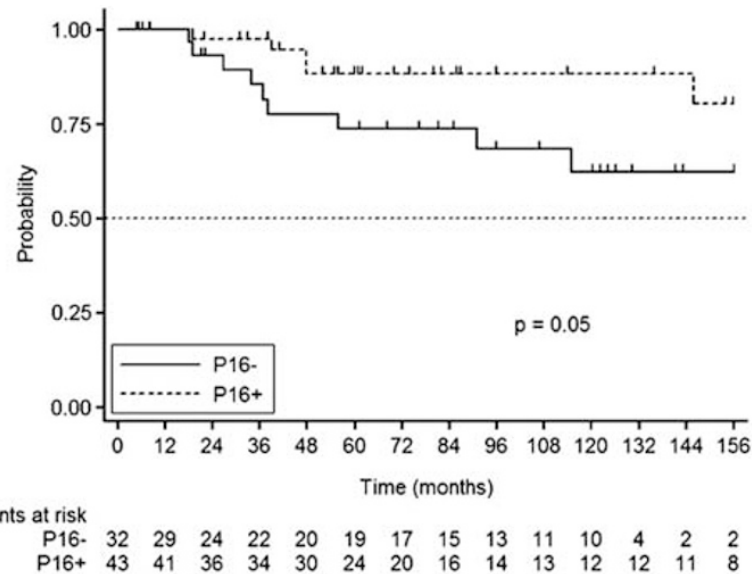

d

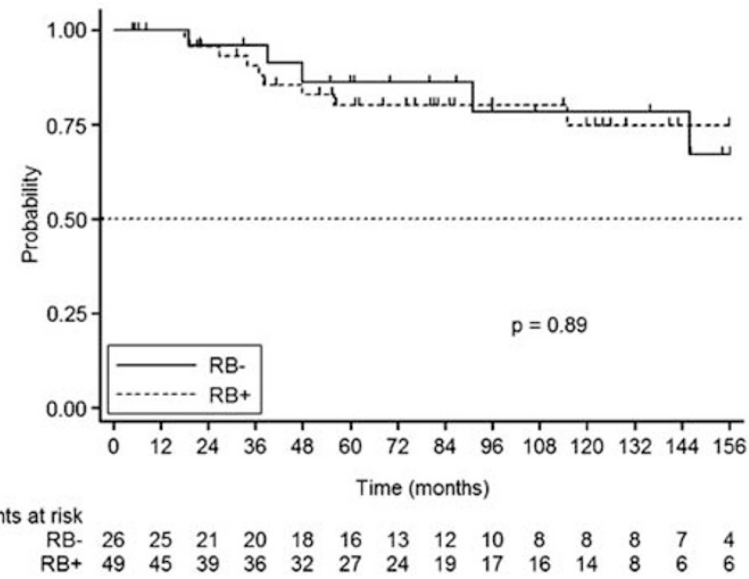

Figure 4 Disease outcome related to p16 and Rb protein expression in patients treated with surgery and chemotherapy. (a) P16 + cases showed a statistical significance for increased disease-free survival. (b) P16 + cases showed a trend towards a statistical significance for increased overall survival. (c and d) $\mathrm{Rb}$ - was not associated with disease-free survival (c) and overall survival (d).

generally devoid of Rb immunostaining. P16 + was correlated with high-grade tumour, high Ki-67, p53 overexpression and CK5 immunopositivity. Conversely, $\mathrm{Rb}$ - showed no association with any variable analyzed.

Subhawong et al, ${ }^{7}$ in a subset of 33 triple-negative breast carcinomas, did not show significant differences in the $\mathrm{Rb}-/ \mathrm{p} 16+$ phenotype between basal-like (CK5 and/or EGFR immunohistochemistry positivity) and unclassifiable (CK5 and EGFR immunohistochemistry negativity) triple-negative breast carcinomas, nor significant correlation between $\mathrm{Rb}-/ \mathrm{p} 16+$ phenotype and p53 overexpression. As shown in our study, the author demonstrated the correlation between $\mathrm{Rb}-/ \mathrm{p} 16+$ phenotype and higher Ki-67 index both in basal-like and unclassifiable triple-negative breast carcinomas.

We demonstrated that patients with p16 + tumors had a better response to adjuvant chemotherapy than patients with p16-: Kaplan-Meier analysis showed a statistically significant increased diseasefree survival $(P=0.001)$ and a trend towards a statistical significance for increased overall survival $(P=0.056)$ (Figures $4 \mathrm{a}$ and $\mathrm{b})$. In multivariate Cox proportional hazards regression analysis, p16 + was independently associated with disease-free survival and overall survival, with a hazard ratio of 0.18 (95\% CI: $0.06-0.51 ; P=0.001)$ and 0.21 (95\% CI: $0.06-0.74 ; P=0.015$ ), respectively (Table 4). Instead, in patients treated with surgery only, prognosis was not affected by p16 immunostaining level (Figures $3 \mathrm{a}$ and b). Conversely, Rb immunostaining level was not associated with clinical outcome, neither in patients treated with surgery only nor in patients treated with adjuvant chemotherapy (Figures 3c, d and $4 \mathrm{c}, \mathrm{d}$ ).

There are many studies showing significant association between $\mathrm{Rb}$ pathway loss or its immunohistochemistry surrogates and clinical outcome in breast cancers, but these studies are irrespective of hormone receptors status, or divided into positive and negative hormone receptors, but irrespective of HER2 status. ${ }^{13,14,16,18,20,25,31}$ However, only few studies focused this issue in triple-negative breast carcinomas.

Arima et $a l^{24}$ showed, in a subset of 60 triple-negative breast carcinomas patients treated with neoadjuvant chemotherapy, that p16 strong immunostaining 
Table 4 Multivariate analysis of predictors of the disease-free survival and overall survival time in no special type TNBCs patients, treated with chemotherapy

\begin{tabular}{|c|c|c|c|}
\hline Factor & $H R$ & $95 \% C I$ & $\mathrm{P}$-value \\
\hline \multicolumn{4}{|c|}{ Disease-free survival } \\
\hline \multicolumn{4}{|c|}{ P16 protein expression } \\
\hline p16- & 1.00 & & \\
\hline $\mathrm{p} 16+$ & 0.18 & $0.06-0.51$ & 0.001 \\
\hline \multicolumn{4}{|c|}{ Nodes metastases } \\
\hline Negative & 1.00 & & \\
\hline Positive & 3.75 & $1.49-9.41$ & 0.005 \\
\hline \multicolumn{4}{|c|}{ Overall survival } \\
\hline \multicolumn{4}{|c|}{ P16 protein expression } \\
\hline p16 - & 1.00 & & \\
\hline $\mathrm{p} 16+$ & 0.21 & $0.06-0.74$ & 0.015 \\
\hline \multicolumn{4}{|c|}{ Tumor size (mm) } \\
\hline$\leq 20$ & 1.00 & & \\
\hline$>20$ & 4.41 & $1.29-15.01$ & 0.018 \\
\hline \multicolumn{4}{|c|}{ Vascular invasions } \\
\hline Absent & 1.00 & & \\
\hline Present & 3.04 & $0.95-9.70$ & 0.060 \\
\hline
\end{tabular}

Abbreviations: CI, confidence interval; HR, hazard ratio; TNBCs, triple-negative breast carcinomas.

was significantly related to chemotherapy response, as reflected by complete pathological response. However, in the same patients, p16 strong immunostaining was not associated with disease-free survival or overall survival in log-rank analysis. Interestingly, the authors demonstrated biological differences between p16 + and p16 - basal-like breast carcinoma cells, with depletion of p16 increasing the percentage of CD44+/CD24 - stem-like cancer cells and reducing chemosensitivity. The reduction of cell proliferation may be a reason for the chemoresistance of p16-depleted cells.

Treré et a ${ }^{10}$ demonstrated, in a subset of 24 triplenegative breast carcinomas, that patients lacking $\mathrm{Rb}$ immunohistochemistry expression had a favorable clinical outcome if treated with adjuvant therapy.

In both these studies, ${ }^{10,24}$ patient selection was different compared with ours: they considered triple-negative breast carcinomas as the tumors with hormone receptors $<10 \%$ and they did not exclude special histotypes (about $20 \%$ of our cases). In addition, having analyzed HER2 status with either immunohistochemistry and silver in situ hybridization methods, we excluded cases HER2 $0 / 1+$ immunohistochemistry but silver in situ hybridization amplified (4.4\% of our cases).

We chose $\geq 1 \%$ as the threshold for hormone receptors positivity because the ASCO-CAP panel recommended considering endocrine therapy in patients whose breast tumor shows at least 1\% ERpositive cells. Also, we think it is very important to select between different histotype of triple-negative breast carcinomas, because of distinct prognostic implications that may be derived; for example, adenoid-cystic and medullary carcinomas and the low-grade apocrine and metaplastic carcinomas are associated with a better prognosis., ${ }^{1,4,6}$ As stated by Montagna et al, ${ }^{6}$ the identification of specialtype triple-negative breast carcinomas has a significant clinical utility and should be considered in therapeutic algorithm. HER2 immunohistochemistry-negative/FISH-positive cases have been reported in a percentage varying from 0 to $3.2 \%, 32$ the reason why we considered necessary to determine ERBB2 gene amplification in tumors 0 or $1+$ immunohistochemistry to avoid inclusion of false triple-negative breast carcinomas cases.

In conclusion, at the best of our knowledge, this is the first study demonstrating that, in a well-defined subset of no special-type triple-negative breast carcinomas, patients with p16 strong immunostained tumors had a good response to adjuvant chemotherapy and p16 immunoexpression can be considered the best surrogate marker for Rb pathway loss.

\section{Acknowledgements}

This work is dedicated to the memory of Marco Venturini.

\section{Disclosure/conflict of interest}

The authors declare no conflict of interest.

\section{References}

1 Carey L, Winer E, Viale G, Cameron D, Gianni L. Triplenegative breast cancer: disease entity or title of convenience? Nat Rev Clin Oncol 2010;7:683-692.

2 Dawson SJ, Provenzano E, Caldas C. Triple negative breast cancers: clinical and prognostic implications. Eur J Cancer 2009;45(Suppl 1):27-40.

3 Linn SC, Van't Veer LJ. Clinical relevance of the triplenegative breast cancer concept: genetic basis and clinical utility of the concept. Eur J Cancer 2009; 45(Suppl 1):11-26.

4 Viale G, Bottiglieri L. Pathological definition of triple negative breast cancer. Eur J Cancer 2009; 45(Suppl 1):5-10.

5 McClendon AK, Dean JL, Rivadeneira DB, et al. CDK4/ 6 inhibition antagonizes the cytotoxic response to anthracycline therapy. Cell Cycle 2012;11:2747-2755.

6 Montagna E, Maisonneuve $\mathrm{P}$, Rotmensz $\mathrm{N}$, et al. Heterogeneity of triple-negative breast cancer: histologic subtyping to inform the outcome. Clin Breast Cancer 2013;13:31-39.

7 Subhawong AP, Subhawong T, Nassar H, et al. Most basal-like breast carcinomas demonstrate the same $\mathrm{Rb}-/ \mathrm{p} 16+$ immunophenotype as the HPV-related poorly differentiated squamous cell carcinomas which they resemble morphologically. Am J Surg Pathol 2009;33:163-175. 
8 Knudsen ES, Knudsen KE. Tailoring to RB: tumour suppressor status and therapeutic response. Nat Rev Cancer 2008;8:714-724.

9 Herschkowitz JI, He X, Fan C, Perou CM. The functional loss of the retinoblastoma tumour suppressor is a common event in basal-like and luminal B breast carcinomas. Breast Cancer Res 2008;10:R75.

10 Treré D, Brighenti E, Donati G, et al. High prevalence of retinoblastoma protein loss in triple-negative breast cancers and its association with a good prognosis in patients treated with adjuvant chemotherapy. Ann Oncol 2009;20:1818-1823.

11 Bosco EE, Knudsen ES. RB in breast cancer: at the crossroads of tumorigenesis and treatment. Cell Cycle 2007;6:667-671.

12 Bosco EE, Wang Y, Xu H, et al. The retinoblastoma tumor suppressor modifies the therapeutic response of breast cancer. J Clin Invest 2007;117:218-228.

13 Ertel A, Dean JL, Rui H, et al. RB-pathway disruption in breast cancer: differential association with disease subtypes, disease-specific prognosis and therapeutic response. Cell Cycle 2010;9:4153-4163.

14 Witkiewicz AK, Ertel A, McFalls J, et al. RB-pathway disruption is associated with improved response to neoadjuvant chemotherapy in breast cancer. Clin Cancer Res 2012;18:5110-5122.

15 Anderson JJ, Tiniakos DG, McIntosh GG, et al. Retinoblastoma protein in human breast carcinoma: immunohistochemical study using a new monoclonal antibody effective on routinely processed tissues. J Pathol 1996;180:65-70.

16 Derenzini M, Donati G, Mazzini G, et al. Loss of retinoblastoma tumor suppressor protein makes human breast cancer cells more sensitive to antimetabolite exposure. Clin Cancer Res 2008;14:2199-2209.

17 Dublin EA, Patel NK, Gillett CE, et al. Retinoblastoma and p16 proteins in mammary carcinoma: their relationship to cyclin D1 and histopathological parameters. Int J Cancer 1998;79:71-75.

18 Lehn S, Ferno M, Jirstrom K, Ryden L, Landberg G. A non-functional retinoblastoma tumor suppressor (RB) pathway in premenopausal breast cancer is associated with resistance to tamoxifen. Cell Cycle 2011;10:956-962.

19 Pietilainen T, Lipponen P, Aaltomaa S, et al. Expression of retinoblastoma gene protein $(\mathrm{Rb})$ in breast cancer as related to established prognostic factors and survival. Eur J Cancer 1995;31A:329-333.

20 Waltersson MA, Askmalm MS, Nordenskjold B, et al. Altered expression of cyclin E and the retinoblastoma protein influences the effect of adjuvant therapy in breast cancer. Int J Oncol 2009;34:441-448.

21 Lakhani SR, Ellis IO, Schnitt SJ, Tan PH, van de Vijver MJ (eds). WHO Classification of Tumours of the Breast. IARC: Lyon 2012.

22 Elston CW, Ellis IO. Pathological prognostic factors in breast cancer. I. The value of histological grade in breast cancer: experience from a large study with longterm follow-up. Histopathology 1991;19:403-410.

23 Witkiewicz AK, Rivadeneira DB, Ertel A, et al. Association of RB/p16-pathway perturbations with DCIS recurrence: dependence on tumor versus tissue microenvironment. Am J Pathol 2011;179: 1171-1178.

24 Arima Y, Hayashi N, Hayashi H, et al. Loss of p16 expression is associated with the stem cell characteristics of surface markers and therapeutic resistance in estrogen receptor-negative breast cancer. Int J Cancer 2011;130:2568-2579.

25 Somlo G, Chu P, Frankel P, et al. Molecular profiling including epidermal growth factor receptor and p21 expression in high-risk breast cancer patients as indicators of outcome. Ann Oncol 2008;19:1853-1859.

26 Schmitt FC, Soares R, Cirnes L, Seruca R. P53 in breast carcinomas: association between presence of mutation and immunohistochemical expression using a semiquantitative approach. Pathol Res Pract 1998;194: 815-819.

27 Livasy CA, Karaca G, Nanda R, et al. Phenotypic evaluation of the basal-like subtype of invasive breast carcinoma. Mod Pathol 2006;19:264-271.

28 Nielsen TO, Hsu FD, Jensen K, et al. Immunohistochemical and clinical characterization of the basal-like subtype of invasive breast carcinoma. Clin Cancer Res 2004;10:5367-5374.

29 Poznic M. Retinoblastoma protein: a central processing unit. J Biosci 2009;34:305-312.

30 Gauthier ML, Berman HK, Miller C, et al. Abrogated response to cellular stress identifies DCIS associated with subsequent tumor events and defines basal-like breast tumors. Cancer Cell 2007;12:479-491.

31 Stengel KR, Dean JL, Seeley SL, Mayhew CN, Knudsen ES. RB status governs differential sensitivity to cytotoxic and molecularly-targeted therapeutic agents. Cell Cycle 2008;7:1095-1103.

32 Sauter G, Lee J, Bartlett JM, Slamon DJ, Press MF. Guidelines for human epidermal growth factor receptor 2 testing: biologic and methodologic considerations. J Clin Oncol 2009;27:1323-1333. 